\title{
Edge Detection in Ventriculograms Using Support Vector Machine Classifiers and Deformable Models
}

\author{
Antonio Bravo ${ }^{1}$, Miguel Vera ${ }^{2}$, and Rubén Medina ${ }^{3}$ \\ ${ }^{1}$ Grupo de Bioingeniería, Universidad Nacional Experimental del Táchira, \\ Decanato de Investigación, San Cristóbal 5001, Venezuela \\ abravo@unet.edu.ve \\ ${ }^{2}$ Laboratorio de Física, Departamento de Ciencias, Universidad de Los \\ Andes-Táchira, San Cristóbal 5001, Venezuela \\ ${ }^{3}$ Grupo de Ingeniería Biomédica (GIBULA), Universidad de Los Andes, \\ Facultad de Ingeniería, Mérida 5101, Venezuela
}

\begin{abstract}
In this paper a left ventricle (LV) contour detection method is described. The method works from an approximate contour defined by anatomical landmarks extracted using Support Vector Machine (SVM) classifiers. The LV contour approximation is used as an initialization step for the deformable model algorithm. An optimization method based on a gradient descend algorithm is used to obtain the optimal contour that provides a minimum energy value. Both classifier and edge detection method performances have been validated. The error is determined as the difference between the shape estimated by the algorithm and the shape traced by an expert.
\end{abstract}

Keywords: anatomical landmarks, left ventricle, support vector machines, edge detection, deformable models.

\section{Introduction}

Segmentation and contour extraction are fundamental tasks in high-level image analysis. The main goal is to divide an image into parts that have a strong correlation with objects or real shapes contained in the image [1.

In cardiac medical imaging modalities, anatomical boundaries cannot be detected by algorithms that use only edge or region information. Low contrast, noise, and non-uniformity of regional intensities are some of the problems associated with cardiac imaging modalities. These problems are always present in ventriculograms. The left ventricle boundary detection in $\mathrm{X}$-ray ventriculograms is a rather complicated task because of the presence of fuzzy superposition of anatomical structures [2].

The aim of this paper is to develop a method combining both SVM and deformable model approaches for LV contour detection. 


\subsection{Support Vector Machines}

Support vector machine is a methodology based on the Vapnik-Chenovenkis learning theory and the structural risk minimization principle [3]. SVMs are efficient non-parametric classification and regression tools [45]. In classification problems SVMs are used for constructing a discriminant function to separate classes using vectors nearest to the decision boundary. The examples or training set for a two-class classification problem can be represented as: $\mathcal{S}=\left\{\left(\mathbf{x}_{i}, y_{i}\right)\right\}_{i=1}^{l} \subset \mathbb{R}^{N} \times\{+1,-1\}$.

The classification task (from $\mathcal{S}$ ) addresses the general problem of finding a discrimination function defined from an input space $\mathbb{R}^{N}$ into an unordered set of classes $\{+1,-1\}$. This discrimination function in some $m$-dimensional feature space is a separating hyper-plane expressed as follows:

$$
f(\mathbf{x})=\operatorname{sign}(\mathbf{w} \cdot \mathbf{x}+b),
$$

where $\mathbf{w}$ is normal to the hyper-plane, $b$ is the bias, $\|\mathbf{w}\|$ is the Euclidean norm of $\mathbf{w}$, and $|b| /\|\mathbf{w}\|$ is the perpendicular distance from the origin to the hyper-plane.

The SVM objective is to find the hyper-plane with minimum norm $\|\mathbf{w}\|^{2}$. The classification problem using linear machines trained on non-separable examples [6]7, can be formulated as a quadratic programming problem where the optimal solution is obtained using Lagrange Multipliers. This solution can be written as:

$$
\mathbf{w}=\sum_{i=1}^{l} \lambda_{i} y_{i} \mathbf{x}_{i}
$$

where $\left\{\lambda_{1}, \ldots, \lambda_{l}\right\}$ are positive Lagrange multipliers. In (2), the examples for which $\lambda_{i}>0$ are known as support vectors and correspond to the critical elements of the training set. In real classification tasks a linear SVM is not appropriate because the classes are generally separated by a non-linear function [7]. In this case, the examples are projected to a feature space of higher (possibly infinite) dimensions via a nonlinear mapping function $\boldsymbol{\Phi}(\cdot)$. This projection process is applied to transform the non-linear problem in the $N$-space to a linear problem in the $M$-space. The SVM solution can be written as:

$$
f(\mathbf{x})=\operatorname{sign}\left(\sum_{i=1}^{l} \lambda_{i} y_{i} \boldsymbol{\Phi}\left(\mathbf{x}_{i}\right) \cdot \boldsymbol{\Phi}(\mathbf{x})+b\right) .
$$

\subsection{Deformable Models}

A deformable model is a parametric contour $\mathcal{C}$ located in the image plane $(u, v) \in$ $\mathbb{R}^{2}$. This contour could be represented as a controlled continuity spline expressed as $\mathbf{p}(s)=(\mathbf{u}(s), \mathbf{v}(s))^{\top}$, where $\mathbf{u}$ and $\mathbf{v}$ are vectors of coordinate functions and $s \in[0,1]$ is the parametric domain [8]. The left ventricle shape defined by contour $\mathcal{C}$ is modeled by the following functional:

$$
\mathcal{E}(\mathbf{p})=\mathcal{I}(\mathbf{p})+\mathcal{P}(\mathbf{p})+\mathcal{R}(\mathbf{p}) .
$$


This functional represents the contour energy that includes the internal energy $\mathcal{I}(\mathbf{p})$, the image energy $\mathcal{P}(\mathbf{p})$ and the external energy $\mathcal{R}(\mathbf{p})$. The optimal contour shape corresponds to the minimum of this energy function. The internal deformation energy (5) incorporates the smoothness and bending properties of the contour.

$$
\mathcal{I}(\mathbf{p})=\int_{s} \alpha(s)\left|\frac{\partial \mathbf{p}}{\partial s}\right|^{2}+\beta(s)\left|\frac{\partial^{2} \mathbf{p}}{\partial s^{2}}\right|^{2} d s,
$$

where $\alpha(s)$ and $\beta(s)$ are weighting coefficients for the smoothness and bending terms respectively. The functional representing the image energy (6) is generally based on the edge detection theory. Several approaches use the theory proposed by Marr and Hildreth [9], where the intensity changes can be detected by finding the maximum or the minimum of $\mathbf{G}_{\sigma} * \mathbf{I}$ that represents the convolution of an image $\mathbf{I}$ with a bi-dimensional Gaussian kernel $\mathbf{G}_{\sigma}$. The image energy pushes the deformable model toward the contour that constitutes the target features.

$$
\mathcal{P}(\mathbf{p})=-\left|\nabla \mathbf{G}_{\sigma} * \mathbf{I}(\mathbf{p})\right|,
$$

where $\sigma$ is the spread parameter (standard deviation) of the Gaussian kernel.

The energy constraints $\mathcal{R}$ imposes additional external forces leading to the minimum energy corresponding to the contour. Different approaches for defining the external constraint forces have been reported by Kass [8].

\section{Method}

\section{$2.1 \quad$ Initialization}

The proposed method uses the traditional deformable contour model for detection of the left ventricle boundary. This deformable model uses a parametric contour $\mathbf{p}(s)$ (see section 1.2) as the input. This parametric contour is deformed in order to minimize the energy functional (4). The deformable model is very sensitive to initialization. Therefore, selecting the initial contour is a very important stage.

Our initial contour is estimated from myocardial landmarks extracted using an approach based on machine learning [10. This approach uses support vector machines (SVM) to localize left ventricle landmarks in ventriculographic sequences. The apex (AP), the basal regions (BA2, BP3, BP4) and the aortic valve sides (VA, VP) are selected as landmarks of interest to construct the SVM classifier (see Figure 1).

A SVM classifier is constructed using the Gaussian Radial Basis Function as parametric kernel. The MatLab Support Vector Machines library is used for performing the training based on a set of 1500 patterns.

SVM Training. Each LV landmark is a $31 \times 31$ pixel pattern manually traced by a cardiologist. A total of 300 patterns constitutes the landmarks dataset (50 patterns for each landmark). A similar procedure is used for obtaining a dataset of 1200 non-landmark pixel patterns generated from angiographic images without 


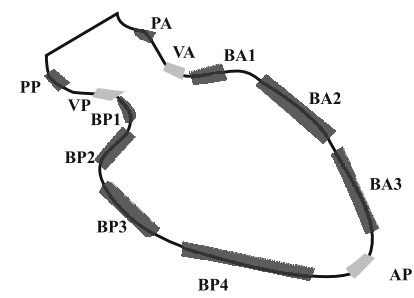

Fig. 1. Fifteen anatomical landmarks established by the American Heart Association

including any landmark information. The training process is used to construct a decision surface. This surface enables classification of input pixel patterns as left ventricle landmarks or non-landmarks. During the test phase a set of 116 images not included in the training set was used. A landmark recognition rate of $98.35 \%$ was obtained.

SVM Based Landmark Detection. The left ventricle approximate border is constructed from landmark points extracted by the SVM classifier. Given the input ventriculographic image, landmarks are located by exhaustively scanning the image for landmark-like patterns. Landmark localization is performed using a $31 \times 31$ sliding window whose content is analyzed by the SVM to determine whether a landmark is present or not. Each landmark is identified by the center point of the $31 \times 31$ pattern considered. After all landmark points are identified, they are joined clockwise starting from VA landmark point and ending in the VP landmark point. Identification of the VA landmark is performed using prior knowledge about the upper part of the aortic valve localization in ventriculographic images.

Initial Contour Estimation. Five more points are estimated using a linear interpolation method from the previously calculated points (AP, BA2, BP3, BP4, VA, VP). The five new points are obtained as follows: the midpoint of the line described by VA and BA2 landmarks is computed. This midpoint is used to construct a new line perpendicular to the line described by VA and BA2 landmarks. The maximum image gradient is searched over this perpendicular line, and the first new point of the set is the one located at the maximum gradient along the line. The remaining points are obtained following the same process between the corresponding pair of landmarks: BA2-AP, AP-BP4, BP4BP3, and BP3-VP. At the end, a new set of eleven points is available to describe the initial LV contour. This set of points is used to generate the parameterized contour using the b-spline method 11. A final discrete set of evenly distributed points is determined by re-sampling the parameterized contour providing the initial contour for the deformable model.

\subsection{Edge Enhancement}

The approaches based on gradient have been widely used for edge enhancement 912 while smoothing filters have been used for minimizing noise content. We 
propose four different techniques to enhance the edges of the left ventricle cavity in the angiographic images. These techniques are based on four smoothing filters: 1) the averaging filter [13, 2) the Gaussian filter [2, 3) the similarity filter [14, and 4) the top-hat morphological filter [15. Once smoothed, the images are processed using an optimal gradient operator [16]. The filters are applied after the initial contour detection. The smoothing filters are applied to the input image, the gradient operator is applied to the smoothed images.

The averaging filter. According to this filter, if a pixel value in the input image $\left(\mathbf{I}_{\text {in }}\right)$ is greater than the average of its neighbors plus a certain threshold $\varepsilon$, then the pixel value in the output image $\left(\mathbf{I}_{\text {average }}\right)$ is set to the average value, otherwise the output pixel is set to the pixel value in the input image. The threshold value $\varepsilon$ was set to the standard deviation of the input image.

The Gaussian filter. Gaussian filtering is a frequently used technique for image smoothing. Each pixel value in the output image $\left(\mathbf{I}_{\text {Gauss }}\right)$ is the result of the convolution between the input image $\left(\mathbf{I}_{\text {in }}\right)$ and a kernel that represents a $2-\mathrm{D}$ Gaussian distribution.

The Similarity filter. This filter quantifies the difference between the graylevel values of pixels in the original image $\mathbf{I}_{\text {in }}$ and in the smoothed image ( $\mathbf{I}_{\text {average }}$ ) based on a similarity criterion [14]. The similarity filter is constructed using the procedure proposed in [17]:

- For each $p_{\mathbf{I}_{\mathbf{i n}}}(i, j) \in \mathbf{I}_{\text {in }}$ and each $p_{\mathbf{I}_{\text {average }}}(i, j) \in \mathbf{I}_{\text {average }}$ obtain the feature vectors $\mathbf{p v}_{\mathbf{I}_{\mathbf{i n}}}=\left[I_{1}, a\right]$ and $\mathbf{p v}_{\mathbf{I}_{\text {average }}}=\left[I_{2}, b\right]$. Where, $I_{1}$ and $I_{2}$ denote the intensities associated with pixel $(i, j)$ and, $a$ and $b$ are the intensity average in a $l \times l$ neighborhood around the pixel $(i, j)$.

- The filter output or similarity image $\left(\mathbf{I}_{\mathrm{S}}\right)$ is obtained according to equation (7).

$$
\mathbf{I}_{\mathrm{S}}=\omega_{1}\left(I_{1}-I_{2}\right)^{2}+\omega_{2}\left(I_{1}-b\right)^{2}+\omega_{3}\left(I_{2}-a\right)^{2},
$$

where $\omega_{1}, \omega_{2}$ and $\omega_{3}$ were set to one.

The top-hat morphological filter. The top-hat is a gray-level morphological operator that can be used as a nonlinear filtering technique. These filters preserve the location of the border transition, and at the same time denoise the image. We consider the white top-hat (WTH) and its dual, the black top-hat (BTH). The white top-hat is defined as the difference between the average image and the opened Gaussian filtered image. The black top-hat is obtained by subtracting the average image from the closed Gaussian filtered image. A disk structuring element (D) of size $11 \times 11$ is used for both the opening and closing operators. Opening (o) and closing (•) morphology operators are derived from the basic operations of erosion $(\ominus)$ and dilation $(\oplus)$ [18. The top-hat images $\mathbf{I}_{\mathrm{BTH}}$ and $\mathbf{I}_{\mathrm{WTH}}$ are calculated according to equation (8).

$$
\mathbf{I}_{\mathrm{BTH}}=\mathbf{I}_{\text {average }}-\left(\mathbf{I}_{\text {Gauss }} \bullet \mathrm{D}\right), \quad \mathbf{I}_{\mathrm{WTH}}=\mathbf{I}_{\text {average }}-\left(\mathbf{I}_{\text {Gauss }} \circ \mathrm{D}\right) .
$$

The optimal gradient operator. These operators can be constructed using a consistency criterion as proposed in 16 . The optimum $5 \times 5$ operator $\left(\nabla_{5 \times 5}\right)$ 
developed by Ando [16, p. 258] is used to obtain $x$ - and $y$-directional gradients associated with the smoothed images.

\subsection{Left Ventricle Deformable Model}

Shape model. The shape model used in our approach is a bi-dimensional dynamic parametric contour $\mathcal{C}$. The smoothness constraint force in the shape model is represented by the internal energy term $\mathcal{I}(\mathbf{p})$. In this paper, we use the internal energy formulation proposed by Kass [8], which is related to the local contour curvature. The internal energy is represented by a linear combination of smoothness and bending contour properties as expressed in (5). This energy term is discretized using the finite difference scheme.

The image energy term $\mathcal{P}$, in the contour energy functional (9), is defined as the average of the gradient magnitude for the smoothed images.

$$
\mathcal{P}(\mathbf{p})=-\gamma \frac{1}{3}\left(\left\|\nabla \mathbf{I}_{\text {Gauss }}(\mathbf{p})\right\|+\left\|\nabla \mathbf{I}_{\mathrm{WTH}}(\mathbf{p})\right\|+\left\|\nabla \mathbf{I}_{\mathrm{BTH}}(\mathbf{p})\right\|\right),
$$

where $\nabla$ represents the optimum gradient operator $\nabla_{5 \times 5}$ proposed in [16] and $\gamma$ is a weighting constant.

Our deformable model incorporates a regularization term $\mathcal{R}$ for attracting the deformable model towards the cardiac cavity edge. The restriction term $\mathcal{R}$ allows to reorient the direction field associated with the image energy term. The restriction is defined from the similarity image obtained using (7). This functional is expressed according to (10) where $\kappa$ is a weighting constant.

$$
\mathcal{R}(\mathbf{p})=-\kappa\left\|\nabla \mathbf{I}_{\mathrm{S}}(\mathbf{p})\right\|
$$

Shape evolution. The dynamics of this model is established according to deformable model theory (see Section [1.2). In this kind of models, the energy functional $(\mathcal{E})$ gives rise to forces deforming the model. The LV shape model energy $(\mathcal{E})$ is minimized by using an iterative optimization process based on a gradient descent algorithm. The aim of this iterative optimization process is to express the current contour shape based on the knowledge of the corresponding previous contour energy. The normalized equation is given by (11).

$$
\mathbf{p}^{k+1}=\mathbf{p}^{k}+\delta \frac{\mathcal{E}\left(\mathbf{p}^{k}\right)}{\left\|\mathcal{E}\left(\mathbf{p}^{k}\right)\right\|},
$$

with $\delta$ denoting the gradient descent step size and $k$ the iteration number.

\section{$3 \quad$ Results}

The proposed method has been tested with mono-plane sequences of ventriculographic images that have been acquired from patients using a digital flat-panel $\mathrm{X}$-rays system (Innova ${ }^{\mathrm{TM}} 4150$, General Electric Medical System). These images 
were acquired using the right anterior oblique (RAO $\left.30^{\circ}\right)$ view. Each image has a resolution of $512 \times 512$ pixels. Each pixel is represented with 8 bits.

The SVM classifier was constructed using the Gaussian radial basis function as a parametric kernel. Our SVM anatomical landmarks classifier is constructed using the Least Squares Support Vector Machines library [19]. The support vectors obtained in the training stage are used to construct the decision surface used to detect the LV landmarks in the original image. The proposed approach has been tested with ventriculograms acquired at several instants of the cardiac cycle. In figure 2, results of the LV landmarks extraction approach for the ventriculogram sequences are shown. Validation of the approach is performed by quantifying the difference between the LV landmark location obtained with respect to the LV landmark located by a cardiologist. The average of the errors obtained (mean \pm standard deviation) for five sequences of ventriculograms in the RAO view, including 163 images is $2.17 \mathrm{~mm} \pm 0.93 \mathrm{~mm}$.
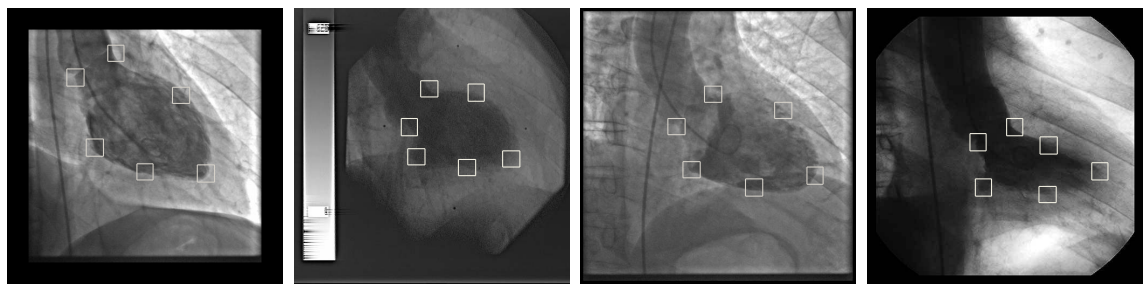

Fig. 2. Bounding white boxes represents the anatomical landmarks obtained

The ventriculographic images are enhanced using the techniques described in section 2.2. Figure 3 shows the enhancement procedure for an end-diastole ventriculogram image. The smoothed image using the Gaussian filter is shown in figure 3. a. Figures 3. b and 3. show the top-hat images (obtained using equation (8) ). The similarity image is shown in figure 3. 3 . Figures 3. 3 , 3. h show the gradient magnitude images obtained from the smoothed images.

The approximate contour is constructed using the procedure described in section 2.1. This approximation is used to initialize our deformable model method. Given the initial contour, the forces associated with the model and its dynamics can be obtained using equations (5), (9), (10) and (11) using the information extracted from preprocessed images as these shown in figure 3. Two of the five sequences analyzed are used to train and initialize the parameter set as follows. The detection process is applied by varying each parameter value. For each parameter, a comparison between the resulting contour and the contour traced by the cardiologist is obtained. The optimal parameter values are $\{\alpha=0.005, \beta=0.00005, \gamma=0.01, \kappa=0.01, \delta=0.5\}$. Figure 4 shows the results of the edge detection process. The image shows a zoom of the LV region where the initial and final contours are located. Figure 4. a shows the approximate contour used to initialize the deformable model. The approximate contour evolution towards the optimum contour is shown in figure $4 \mathrm{~b}$, where the initial contour is 


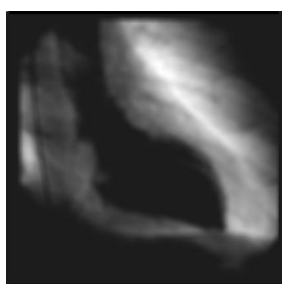

(a)

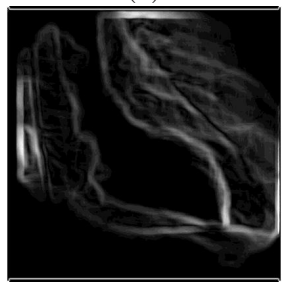

(e)

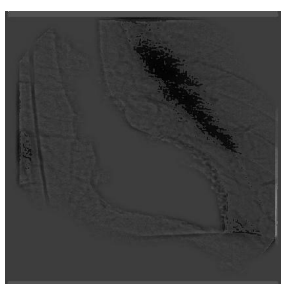

(b)

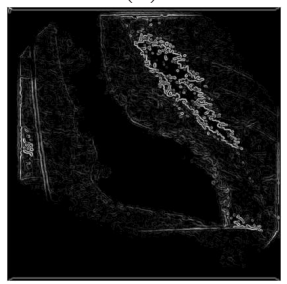

(f)

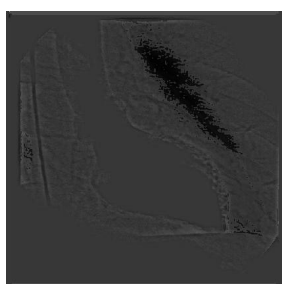

(c)

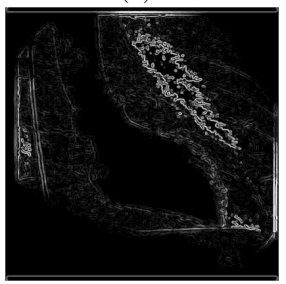

$(\mathrm{g})$

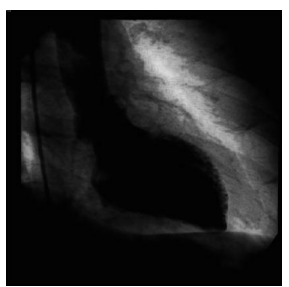

(d)

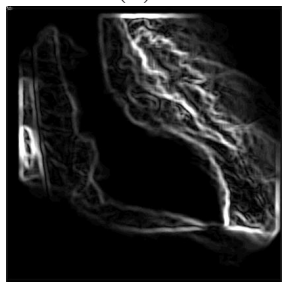

(h)

Fig. 3. Edge enhancement results. a) Gaussian filtered image. b) Black top-hat image. c) White top-hat image. d) Similarity image. e) Gradient magnitude for the Gaussian image. f) Gradient magnitude for the Black top-hat image. g) Gradient magnitude for the White top-hat image. g) Gradient magnitude for the Similarity image.

indicated by white dash-dotted line and the final contour by black dash-dotted line. Figure 4.c shows the contour energy behavior where the minimum energy state is reached after few iterations. Figure 5 shows the results obtained for the end diastole images in four ventriculographic sequences. The contours traced by the expert are shown using white dash-dotted lines and the final segmentations are shown using black dash-dotted lines. The performance of the segmentation method is validated using the approach proposed by Suzuki [20, p. 335]. Five ventriculogram sequences including a total of 163 images are considered during the validation process. Comparison between the segmented contours and the contours traced by a cardiologist shows an average contour error $E_{C}$ of $5.97 \%$ and an average area error $E_{A}$ of $3.71 \%$.

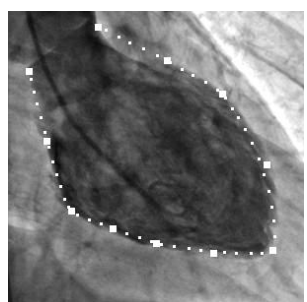

(a)

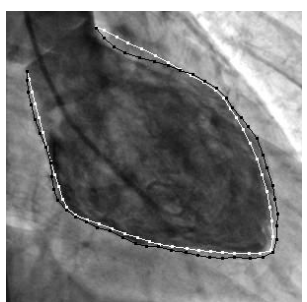

(b)

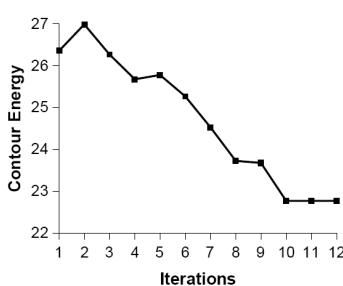

(c)

Fig. 4. Edge detection process. a) Initial contour (white dash-dotted line). c) Evolution to final contour (black dash-dotted line). c) Contour energy evolution. 

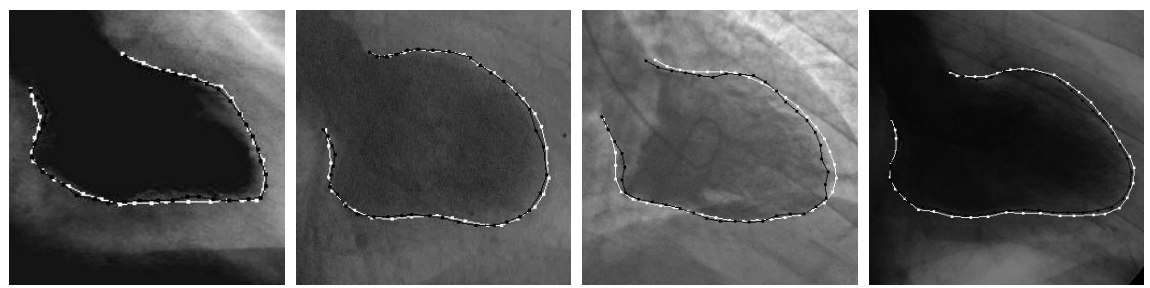

Fig. 5. Results of the left ventricle segmentation. Ground truth contour indicated by a white dash-dotted line. Contour extracted by the proposed approach is shown using a black dash-dotted line.

\section{Conclusions}

This paper has presented an automatic method for segmentation of the LV shape using SVM and deformable models. The accurate initialization of the deformable model is performed based on landmarks extracted using Support Vector Machine classifiers. The SVM classifier approach does not require preprocessing of the input data. The deformable model incorporates information about the ventricular edge by means of an energy functional expressed as a linear combination of the gradient magnitude estimated from several edge enhanced images.

The proposed segmentation method is accurate for automatically detecting the left ventricle contour in ventriculograms. The accuracy has been accessed by tests performed for the SVM based initialization approach as well as the validation for the complete segmentation method. Further research is aimed at using a multi-class SVM and at performing a more complete validation.

Several efforts to develop an automatic contour detection method for ventricular images have been reported so far [20121. However, these methods have not been subjected to an extensive clinical validation. In this paper, we have proposed an alternative automatic method that can be implemented easily. It enables the quantitative analysis of the cardiovascular function based on ventriculograms.

\section{Acknowledgments}

The authors would like to thank the Investigation Dean's Office of Universidad Nacional Experimental del Táchira, CDCHT from Universidad de Los AndesTáchira and FONACIT grant G-2005000342 for their support to this project.

\section{References}

1. Sui, L., Haralick, R., Sheehan, F.: A knowledge-based boundary delineation system for contrast ventriculograms. IEEE Trans. Inform. Technol. Biomed. 5(2), 116-132 (2001)

2. Pratt, W.: Digital Image Processing. John Wiley - Sons, USA (1978)

3. Vapnik, V.: The Nature of Statistical Learning Theory. Springer, New York (1995) 
4. Osuna, E., Freund, R., Girosi, F.: Training support vector machines: an application to face detection. In: CVPR 1997, San Juan, Puerto Rico, pp. 130-136 (1997)

5. Smola, A.J.: Learning with Kernels. PhD thesis, Technische Universit

6. Burges, C.: A tutorial on support vector machines for pattern recognition. Knowledge Discovery and Data Mining 2(2), 121-167 (1998)

7. Osuna, E., Freund, R., Girosi, F.: Support vector machines: Training and applications. Technical report, Artificial Intelligence Laboratory, Massachusetts Institute of Technology (1997)

8. Kass, M., Witkin, A., Terzopoulos, D.: Snakes: Active contours models. Int. J. Comput. Vis. 1, 321-331 (1987)

9. Marr, D., Hildreth, E.: Theory of the edge detection. Proccedings of the Royal Society of London 207, 187-217 (1980)

10. Vera, M., Bravo, A.: Left ventricle image landmarks extraction using support vector machines. In: Proceedings of $2^{\text {nd }}$ International Conference on Computer Vision Theory and Applications, Barcelona, Spain, pp. 339-343 (2007)

11. Barsky, B.A.: Computer Graphics and Geometric Modeling Using Beta-Splines. Springer, USA (1988)

12. Canny, J.: A computational approach to edge detection. IEEE Trans. Pattern Anal. Machine Intell. PAMI-8, 679-698 (1986)

13. Gonzalez, R., Woods, R.: Digital Image Processing. Addison-Wesley Publishing Company, New-Jersey (1992)

14. Haralick, R., Shapiro, L.: Computer and Robot Vision, vol. I. Addison-Wesley Publishing Company, USA (1992)

15. Hanbury, A., Serra, J.: Morphological operators on the unit circle. IEEE Trans. Image Processing 10(12), 1842-1850 (2001)

16. Ando, S.: Consistent gradient operators. IEEE Trans. Pattern Anal. Machine Intell. 22(3), 252-264 (2000)

17. Bravo, A., Medina, R., Diaz, J.A.: A clustering based approach for automatic image segmentation: An application to biplane ventriculograms. In: Martínez-Trinidad, J.F., Carrasco Ochoa, J.A., Kittler, J. (eds.) CIARP 2006. LNCS, vol. 4225, pp. 316-325. Springer, Heidelberg (2006)

18. Serra, J.: Image Analysis and Mathematical Morphology. A Press, London (1982)

19. Suykens, J., Gestel, T.V., Brabanter, J.D., Moor, B.D., Vandewalle, J.: Least Squares Support Vector Machines. World Scientific, Singapore (2002)

20. Suzuki, K., Horiba, I., Sugie, N., Nanki, M.: Extraction of left ventricular contours from left ventriculograms by means of a neural edge detector. IEEE Trans. Med. Imag. 23(3), 330-339 (2004)

21. Oost, E., Koning, G., Sonka, M., Oemrawsingh, P.V., Reiber, J.H.C., Lelieveldt, B.P.F.: Automated contour detection in $\mathrm{x}$-ray left ventricular angiograms using multiview active appearance models and dynamic programming. IEEE Trans. Med. Imag. 25(9), 1158-1171 (2006) 